\title{
Extracellular Expression and Catalytic Bioactivity of Optimized Human Aromatase (CYP19) in Pichia Pastoris
}

\author{
Yan-ying $\mathrm{HU}^{1}$, Yong-hui $\mathrm{ZHONG}^{1}$, Jing $\mathrm{ZHEN}^{2}$, Hui-tu $\mathrm{ZHANG}^{1}$, Shi-ru \\ $\mathrm{JIA}^{1}$, Fu-ping LU ${ }^{1}$, Yu-jie, DAI ${ }^{1, a,{ }^{*}}$ and Xiu-li ZHANG ${ }^{1, b,{ }^{*}}$
}

${ }^{1}$ College of Bioengineering, Tianjin University of Science and Technology, Tianjin 300457, P.R. China

${ }^{2}$ Department of Medicinal Chemistry, School of Environmental and Biological Sciences, Rutgers University, NJ 08901, USA

\author{
ayjdai@126.com \\ bzxl1966@hotmail.com \\ ${ }^{*}$ Corresponding author
}

Keywords: Recombinant aromatase, Pichia pastoris, Heterologous protein production.

\begin{abstract}
The fragment encoding aromatase (CYP19) with $45 \mathrm{~N}$-terminal amino acids cut-off was cloned into pPIC9K vector and transformed to the yeast Pichia pastoris. The multi-copy recombinant strain was selected for the production of recombinant aromatase. The catalytic bioactivity of secreted enzyme was further verified by fluorescent substrate detection method. The results indicate that aromatase was successfully expressed in recombinant Pichia pastoris strain and secreted into the culture supernatant. The extracellular expression of the bioactive aromatase by $P$. pastoris provides us a better alternative for aromatase commercial production and promotes the research progress for the discovery of novel aromatase.
\end{abstract}

\section{Introduction}

Estrogen plays an important role in breast cancer development [1]. After binding to estrogen receptor, it can activate transcription of target genes, which are responsible for cancer cell proliferation in estrogen-dependent breast tumors. The biosynthesis of estrogen from adipose tissue increases along with body weight and age [2]. It has been found that body weight and age increase have good correlation with an increased incidence of postmenopausal breast cancer [3, 4]. The conversion from androgens to estrogens is catalyzed by aromatase (CYP19), a microsomal enzyme that serves as a rate-limiting enzyme in estrogen biosynthesis. Thus, aromatase is an important target for the endocrine therapy of postmenopausal breast cancer. Each year there are many research investigations on aromatase inhibitors and large amount of aromatase is needed for the bioactivity assays. In the early days, the placental microsomal with coarse aromatase were applied for the bioactivity assay. However, other impurities are unavoidable and may influence the experimental results. There is also an ethical issue on the use of placental microsomal from human beings. Therefore, it is important to find alternative way to produce large amount of aromatase with high purity. The expression of gene engineering techniques have been employed for the high throughput aromatase inhibitor screening kit. Although human CYP19 has been expressed in E.coli [5], yeast [6], and insect cells [7-9], only inclusion body was obtained from E.coli expression due to the lack of necessary protein modification system. On the other hand, even if it can be actively expressed in eukaryotic cells, such as yeast and insect cells, the harsh cultivation conditions and low growth rate of the insect cells limit its extensive application for producing aromatase. The low level of intracellular expression and the difficulty of enzyme purification have hampered the application of 
Saccharomyces cerevisiae for the production of aromatase. Comparatively, Pichia pastoris has higher expression efficiency and the enzyme can be more easily collected and purified when the extracellular expression is employed [10]. Thus, in this study, the extracellular expression of human aromatase (CYP19) by Pichia pastoris was investigated. In order to make it convenient to release aromatase to the culture medium, $45 \mathrm{~N}$-terminal amino acids as membrane anchoring sequences were cut off and the bioactivity of the secreted aromatase was assayed by the fluorescent substrate (7- hydroxy-4-trifluoromethyl coumarin. HFC) detection method [11].

\section{Materials and Methods}

\section{Strains and Vectors}

Escherichia coli DH5 $\alpha$ and Pichia pastoris GS115 were used as the host strain for heterogonous expression of aromatase gene. The vector pPIC9K was lab stored and was used as the yeast expression vector.

\section{Medium and Reagents}

LB and YPD culture medium were used for the cultivation of E. coli DH5 $\alpha$ and P. pastoris respectively. Recombinant transformants were screened using MD medium. The heterogonous aromatase were produced in BMGY and BMMY, respectively.The plasmid mini-preps kit and DNA gel extraction kit were purchased from Sangon Biotech (Shanghai, China). Restriction enzymes, T4 DNA polymerase and Pyrobest DNA polymerase were purchased from Takara (Japan). The cDNA of aromatase gene was obtained from GenBank (NM_000103.3), optimized to meet the usage preference of Pichia pastorisand synthesized by Genewiz Biotech (Suzhou, China) and inserted into pUC57 vector.All other reagents used in this work were of analytical grade unless specified.

\section{Construction of Expression Plasmid}

The primers used for aromatase amplification were as follows: 5'-TACGTAAGCATCCCGGGTCCGGGCTAC-3' (up) and 5'-GCGGCCGCTTAATGTTCCAGACAACGATCACTGTTG-3' (down). The aromatase gene that $45 \mathrm{~N}$-terminus residues were cut off was amplified by PCR using plasmid pUC57-aromatase as the template. The PCR product was purified, digested with restriction enzyme SnaB I and Not I and then inserted into pPIC9K to construct the final expression plasmid pPIC9K-aromatase.

\section{Transformation and Selection of Transformants}

The expression plasmid was linearized by Sal I before electroporation into P. pastoris (MicroPulser Electroporator, Bio-Rad, USA) according to Lin-Cereghino [12], which would then be spread on MD plates. YPD containing $0.5 \mathrm{mg} \cdot \mathrm{mL}^{-1}$ and $2 \mathrm{mg} \cdot \mathrm{mL}^{-1} \mathrm{G} 418$ were used to further screen the promising transformants respectively. Selected transformants were cultured in YPD and detected for the presence of aromatase gene in chromosomal DNA by PCR and confirmed by DNA sequencing.

\section{Aromatase Production by Transformants}

Transformants was inoculated into $5 \mathrm{ml} \mathrm{YPD}$ for $16-18 \mathrm{~h}\left(30^{\circ} \mathrm{C}, 180 \mathrm{r} \cdot \mathrm{min}^{-1}\right)$, and $500 \mu \mathrm{l}$ culture was transferred into $50 \mathrm{ml}$ BMGY containing $50 \mu \mathrm{g} \cdot \mathrm{mL}^{-1}$ kanamycin for growth until OD600 reached from 2 to 6 . All cells were harvested by centrifugation and resuspended in BMMY with the initial OD600 was approximately 1.0. The production of aromatase was induced for $60 \mathrm{~h}$ by supplementing $0.5 \%(\mathrm{v} / \mathrm{v})$ methanol every $24 \mathrm{~h}$. The fermentation culture was centrifuged at $8000 \mathrm{r} \cdot \mathrm{min}^{-1}$ for $10 \mathrm{~min}$. The supernatant was 
salted-out overnight at $4{ }^{\circ} \mathrm{C}$. The precipitates were dissolved in phosphate buffer of $\mathrm{pH} 7.4$ and dialyzed, resulted in the crude enzyme solution.

\section{SDS-PAGE Analysis}

Sodium dodecyl sulfate polyacrylamide gel electrophoresis (SDS-PAGE) was performed to analyzed the crude enzyme solution using 12\% acrylamide gel [13]. Followed by electrophoresis, the gel was stained with Coomassie Brilliant Blue R-250 and decolorized overnight. Proteins were visualized on ODYSSEY infrared imaging (Li-cor Biosciences, USA).

\section{Assay of Aromatase Activity}

Aromatase can convert 7-methoxy-4-trifluoromethyl coumarin (MFC) to 7-hydroxy-4-trifluoromethyl coumarin (HFC) [11]. The bioactivity of the recombined aromatase was assayed by the fluorescent substrate method according to the instruction of the CYP19/MFC high throughput inhibitor screening kit (CY-TECH, Beijing, China). Differently, the original mixture of aromatase and cytochrome P450 reductase was substituted by our crude enzyme mixed with cytochrome P450 reductase isolated from porcine liver as described by Yasukochi Y et. Al [14]. In brief, 100 $\mu 1$ NADPH/Cofactor Mix (50mM potassium phosphate buffer (K-P buffer) containing $2.6 \mathrm{mM}$ NADPH, $7.6 \mathrm{mM}$ glucose-6-phosphate, 0.8 unit $\cdot \mathrm{mL}^{-1}$ glucose-6-phosphate dehydrogenase and $1 \mathrm{mg} \cdot \mathrm{mL}^{-1}$ albumin, $\mathrm{pH}=7.4$ ) were incubated for $10 \mathrm{~min}$ at $37^{\circ} \mathrm{C}$ using a 96 -well plate. $100 \mu \mathrm{l}$ Enzyme/Substrate Mix (K-P buffer containing $25 \mathrm{mM}$ MFC, $1 \mu \mathrm{M}$ cytochrome P450 reductase and crude enzyme solution, $\mathrm{pH}=7.4$ ) was added quickly and reacted for $30 \mathrm{~min}$ at $37^{\circ} \mathrm{C}$. The reaction was terminated by $75 \mu 1$ termination reagent $(0.5 \mathrm{M}$ Tris Base $)$ and the mixture was incubated for additional $2 \mathrm{~h}$. The control was prepared as follows: $100 \mu \mathrm{l}$ $\mathrm{NADPH} / \mathrm{Cofactor} \mathrm{Mix}$ was incubated for $30 \mathrm{~min}$ at $37^{\circ} \mathrm{C}$, after the addition of $75 \mu \mathrm{l}$ termination reagent, $100 \mu \mathrm{l}$ Enzyme/Substrate Mix was added. The fluorescence was measured at excitation wave length $409 \mathrm{~nm}$ and absorption wave length 530nm using an Microplate Reader (Thermo, USA). The change of fluorescence can be used to determine the activity of aromatase.

\section{Results and Discussion}

\section{Construction of Expression Plasmids}

Aromatase gene with $45 \mathrm{~N}$-terminus acid cutoff, a 1374 bp nucleotide sequence was amplified by PCR, double digested with SnaB I and Not I, and inserted into vector pPIC9K resulting in a recombinant aromatase-expressing plasmid called pPIC9K-aromatase.

\section{Screening and Verification of Transformants}

Subsequent to linearization with Sal, pPIC9K-aromatase was transformed into Pichia pastoris competent cells by electroporation[12]. The sac I -linearized pPIC9K vector was transformed as control. The transformants were obtained on MD plates, rescreened on YPD plates containing $0.5 \mathrm{mg} \cdot \mathrm{mL}^{-1}$ and $2 \mathrm{mg} \cdot \mathrm{mL}^{-1} \mathrm{G} 418$ for $3 \mathrm{~d}$ at $30^{\circ} \mathrm{C}$ and verified by PCR using the primers in section 2.1. The result revealed that the target aromatase gene was stably integrated into the host cell genome.

\section{Expression of Recombinant Aromatase}

The transformant was incubated in YPD at $30^{\circ} \mathrm{C}$ and $200 \mathrm{r} \cdot \mathrm{min}^{-1}$ for $24 \mathrm{~h}$, and subsequently the cultures were centrifuged and re-suspended in BMMY to induce aromatase expression by $1 \%$ methanol for $60 \mathrm{~h}$, as described in Section 2.6. Aliquots of the fermentation culture 
supernatant were preliminary purified and subsequently analyzed by SDS-PAGE. As shown in Fig. 1, a band of approximately $50 \mathrm{kDa}$ which was similar to the predicted mol wt of recombined aromatase was observed in the supernatant of GS115/pPIC9K-aromatase strain, while no such band was detected in the supernatant of P.pastoris with integrated pPIC9K. The results indicate that aromatase was possibly expressed in P.pastoris strains and secreted into the culture supernatant successfully. The supernatant was used to evaluate the bioactivity of recombinant aromatase.

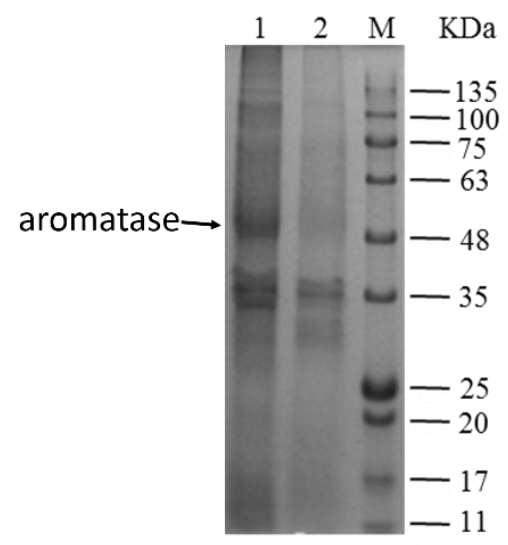

Fig.1 SDS-PAGE analysis of heterologously expressed aromatase. Lane M: The protein molecular weight marker; lane 1: supernatants of recombinant GS115/pPIC9K-aromatase; lane 2: supernatant of negative control strain GS115/ pPIC9K.

\section{Activity Assessment of Recombinant Aromatase}

The bioactivity of recombinant aromatase was evaluated as described as Section 2.8. The activity assessment results are shown in Fig. 2. The production of HFC in group A is $77.1 \pm 1.4 \mathrm{nmol} \cdot \mathrm{L}^{-1}$ while no HFC production were detected in group $\mathrm{B}, \mathrm{C}$ and $\mathrm{D}$, suggests that the recombinant aromatase showed bioactivity. $19.3 \pm 0.35 \mathrm{nmol}$ HFC was yielded in $30 \mathrm{~min}$ per $1 \mathrm{ml}$ aromatase crude enzyme solution at 37 and $\mathrm{pH}$ 7.4.

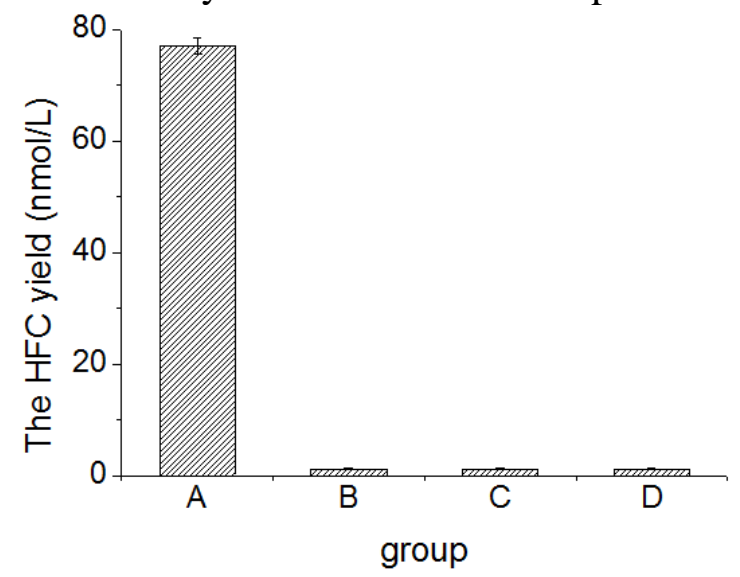

Fig.2Recombinant aromatase catalytic activity. (A) The catalytic activity of crude enzyme solution generated by GS115/pPIC9K-aromatase strain; (B) The catalytic activity of crude enzyme solution generated by GS115/pPIC9K strain; (C) Control to GS115/pPIC9K-aromatase strain; (D) Control to GS115/pPIC9K strain. For $\mathrm{C}$ and D, the Enzyme/Substrate Mix was added followed by the reaction was terminated.

\section{Conclusions}

Due to the simplicity of techniques, efficiency of secretion and AOX strong promoter, $P$. pastorihas become one of the most suitable heterologous protein expression host [15]. It is profitable that the fermentation products transit into extracellular space because cell 
disruption is unrequired and the purification of products extracellular secreted are obviously facilitated. Residues 21-42 of aromatase were protein transmembrane segment and the 45 $\mathrm{N}$-terminus amino acids were membrane-bound polypeptide chain contributed to the close link between aromatase and smooth endoplasmic reticulum[16]. Aromatase without the 38 $\mathrm{N}$-terminus residues was successfully expressed by $E$. coli, and a functionally active aromatase isolated would efficiently catalyzed the aromatization of androgens [17].

In this work, a $45 \mathrm{~N}$-terminus amino acid cut off aromatase gene was optimized and successfully transformed into $P$. pastoris. The multi-copy recombinant strains were screened on YPD plates containing G418 and were induced by $0.5 \%$ methanol to express aromatase. As expected, the target aromatase was detected in the culture supernatant of selected recombinant $P$. pastoris strains, which proved that the extracellular aromatase was successfully expressed. Moreover, the expressed protein showed the aromatase bioactivity converting MFC to HFC. The results indicated that the recombinant aromatase has catalytic properties despite the deletion of $45 \mathrm{~N}$-terminus amino acids. Furthermore, the 45 $\mathrm{N}$-terminus amino acids deletion makes it is easier for the recombinant aromatase secretion into the extracellular. The extracellular expression of the bioactive aromatase by $P$. pastorisgives us a better alternative for aromatase commercial availability and promotes the process for finding novel aromatase.

\section{Acknowledgement}

This work was supported by the National Natural Science Foundation of China (Grant No. 21272171).

\section{References}

[1] Gustafsson, J.-A., M. Warner, Estrogen receptor $\beta$ in the breast: role in estrogen responsiveness and development of breast cancer. J Steroid Biochem. 74 (2000)245-248.

[2] James, V.H.T., M. Serio, et al., The Endocrine function of the human ovary. Proceedings of the Serono Symposia, London: Academic Press. (1976) 135-140.

[3] Mcguire, W.L., A physiological role for estrogen and progesterone in breast cancer, J Steroid Biochem.7 (1976) 875-82.

[4] Siiteri, P.K., Steroid hormones and endometrial cancer,Cancer Res.38 (1978) 4360-4366.

[5] Kagawa, N., Q. Cao, K. Kusano, Expression of human aromatase (CYP19) in Escherichia coli by N-terminal replacement and induction of cold stress response, Steroids.68 (2003) 205-209.

[6] Pompon D, Liu RY, Besman MJ, et al., Expression of human placental aromatase in Saccharomyces cerevisiae,Mol Endocrinol. 9 (1989) 1477-1487.

[7] Lahde M, Raunio H, Pelkonen O, et al., Expression of Human Placental Cytochrome P450 Aromatase (CYP19) cDNA in Insect Cells Using a Luciferase-Based Baculovirus Vector. Biochem Bioph Res Co. 197 (1993) 1511-1517.

[8] Sigle RO, Titus MA, Harada N, et al., Baculovirus Mediated High Level Expression of Human Placental Aromatase (CYP19A1),Biochem Bioph Res Co.201 (1994) 694-700.

[9] Amarneh, B., E.R. Simpson, Expression of a recombinant derivative of human aromatase $\mathrm{P} 450$ in insect cells utilizing the baculovirus vector system,Mol Cell Endocrinol.109 (1995) 1-5. 
[10] Cereghino, J.L., J.M. Cregg, Heterologous protein expression in the methylotrophic yeast Pichia pastoris. Fems Microbiol Rev. 24 (2000) 45-66.

[11] Porrogi P, Kóbori L, Kõhalmy K, et al., Limited applicability of 7-methoxy-4-trifluoromethylcoumarin as a CYP2C9-selective substrate. Pharmacol Rep.60 (2008) 972-979.

[12] Lin-Cereghino J, Wong WW, Xiong S, et al., Condensed protocol for competent cell preparation and transformation of the methylotrophic yeast Pichia pastoris, Biotechniques, 38 (2005) 44, 46, 48.

[13] Zhang X, Li X, Xia L, Heterologous Expression of an Alkali and Thermotolerant Lipase from Talaromyces thermophilus in Trichoderma reesei. Appl Biochem Biotech. 176 (2015) 1722-1735.

[14] Yasukochi Y, Masters BS, Some properties of a detergent-solubilized NADPH-cytochrome c(cytochrome P-450) reductase purified by biospecific affinity chromatography. J Biol Chem. 251 (1976) 5337-5344.

[15] Schmidt, F.R., Recombinant expression systems in the pharmaceutical industry. Appl Microbiol Biot. 65 (2004) 363-372.

[16] Ghosh D, Griswold J, Erman M, et al., Structural basis for androgen specificity and oestrogen synthesis in human aromatase, Nature. 457 (2009) 219-223.

[17] Zhang F, Zhou D, Kao YC, et al., Expression and purification of a recombinant form of human aromatase from Escherichia coli,Biochem Pharmacol.64 (2002) 1317-1324. 